\title{
Medial unicondylar knee arthroplasty should be reserved for patients with complete joint space collapse
}

\author{
Alexander Wurm ${ }^{1} \cdot$ Anna Zechling ${ }^{2} \cdot$ Hermann Leitner $^{3} \cdot$ Dietmar Dammerer $^{1} \cdot$ Bernhard Pfeifer $^{3} \cdot$ Martin Krismer $^{1}$. \\ Michael Liebensteiner ${ }^{1}$
}

Received: 11 February 2021 / Accepted: 20 April 2021 / Published online: 1 May 2021

(c) The Author(s) 2021

\begin{abstract}
Purpose To determine whether preoperative radiologic joint space width (JSW) is related to the outcome of medial unicondylar knee arthroplasty (UKA) (primary hypothesis).

Methods A retrospective comparative analysis was performed. One group was comprised of UKA patients with preoperative JSW 0-1 mm. Another group was made up of patients with preoperative JSW $\geq 2 \mathrm{~mm}$ (range $0-4 \mathrm{~mm}$ ). The JSW was measured from preoperative weight-bearing Schuss-view radiographs. The clinical outcome was determined with the Western Ontario and MacMaster Universities (WOMAC) Osteoarthritis Index score preoperatively and 1 year after medial UKA. Implant survival data were obtained from the arthroplasty register of Tyrol.

Results There were 80 patients with a preoperative JSW 0-1 mm (age 66, BMI 27.8) and 70 patients with a preoperative $\mathrm{JSW} \geq 2 \mathrm{~mm}$ (age 64, IQR 15, BMI 28.1). WOMAC total was $10 \pm 10$ in patients with $0-1 \mathrm{~mm}$ JSW and $25 \pm 47$ in patients with $\geq 2 \mathrm{~mm} \mathrm{JSW}$ at 1 year postoperative $(p=0.052)$. WOMAC pain at 1 year postoperative was $7 \pm 16$ in patients with $0-1 \mathrm{~mm} \mathrm{JSW}$ and $18 \pm 46$ in patients with $\geq 2 \mathrm{~mm}$ JSW $(p=0.047)$. WOMAC function at 1 year postoperative was $10 \pm 9$ in patients with $0-1 \mathrm{~mm} \mathrm{JSW}$ and $17 \pm 51$ in patients with $\geq 2 \mathrm{~mm} \mathrm{JSW}(p=0.048)$. In patients with $0-1 \mathrm{~mm}$ JSW 5 year prosthesis survival was $92.3 \%$ and in patients with $\geq 2 \mathrm{~mm} \mathrm{JSW}$, it was $81.1 \%(p=0.016)$.

Conclusions In patients with preoperative complete joint space collapse (0-1 mm JSW), clinical outcome was superior to that of patients with incomplete joint space collapse. This was true for both 1 year postoperative WOMAC pain and WOMAC function and for 5 year implant survival rates. On the basis of our findings, it is recommended that 'complete joint space collapse' especially be used to achieve best clinical outcome in medial UKA surgery.
\end{abstract}

Level of evidence IV.

Keywords Total knee arthroplasty $\cdot$ Joint space narrowing $\cdot$ Joint space width $\cdot$ Osteoarthritis $\cdot$ Total knee replacement . Outcome

\author{
Abbreviations \\ JSW \\ UKA \\ Joint space width \\ WOMAC Score \\ Unicondylar knee arthroplasty \\ Western Ontario and MacMaster Uni- \\ versities Osteoarthritis Index Score \\ Alexander Wurm \\ alexander.wurm@i-med.ac.at \\ 1 Department of Orthopaedics and Traumatology, Medical \\ University of Innsbruck, Anichstrase 35, 6020 Innsbruck, \\ Austria \\ 2 Medical University of Innsbruck, Innsbruck, Austria \\ 3 Department of Clinical Epidemiology, Tyrolean Federal \\ Institute for Integrated Care, Tirol Kliniken GmbH, \\ Innsbruck, Austria
}

BMI

TKA

KL

$\mathrm{OA}$

PACS
Body mass index

Total knee arthroplasty

Kellgren-Lawrence

Osteoarthritis

Picture Archiving and Communication System

\section{Introduction}

In the field of medial unicondylar knee arthroplasty (UKA), only three studies exist that examined the relationship between preoperative OA severity and clinical outcome [5, 7, 10]. Knifsund et al. investigated 294 UKA cases [7]. They reported that those undergoing UKA with preoperative 
Kellgren-Lawrence (KL) Grade 0-2 had a significantly greater risk for later reoperation than did those with KL Grade 3-4. Knifsund et al. also stated that knees with a joint space width of more than $1 \mathrm{~mm}$ have a greater risk for revision surgery. However, they did not report patient-reported outcome (e.g. knee scores). Maier et al. also investigated the influence of the preoperative stage of OA on UKA outcome [10]. The authors reported on 64 patients and compared cases with partial joint space collapse and those with complete joint space collapse regarding knee scores and implant survival. There were no significant differences in knee score outcome and also no significant differences in revision rates. Hamilton et al. analysed 94 UKA with preoperatively only partial cartilage loss [5]. Outcome in those patients was poorer than in patients with full-thickness cartilage loss: more reoperations, inferior results in Oxford Knee Score. In summary, studies of that topic were rare and the three available studies provided conflicting information.

In light of the shortcomings of previous research, our study approach incorporated: (a) robust implant survival data from the arthroplasty registry of Tyrol, (b) use of a patient-reported outcome score along with implant survival, (c) use of the OA staging method 'joint space width' that was reported to be superior to Kellgren-Lawrence stages.

It was hypothesised that patients without complete radiologic joint space collapse would experience a different clinical knee score outcome (WOMAC score) than would those with complete radiologic joint space collapse (primary hypothesis).

\section{Methods}

A retrospective comparative design was applied. Data already available from clinical routine were analysed after approval by the Ethics Committee of the medical university (approval No. AN2017-0021-370/4.1). Analysed were patients who had previously undergone primary UKA at our department as part of clinical routine. Inclusion/exclusion of patients was handled in accordance with the Oxford surgical manual. In addition, patients were excluded from data analysis in the case of: (a) incomplete WOMAC data, (b) primary prostheses other than medial Oxford UKA, and (c) missing preoperative Schuss-view radiograph.

All surgical procedures were performed as part of our hospital's clinical routine. Patients always underwent the 'Oxford Phase-3' medial UKA (Biomet Inc., Warsaw, Indiana, USA). The surgical technique was as recommended in the manufacturer's surgical manual.

Joint space width was determined from radiographs in the medical university hospital's PACS by always the same investigator using the same software (Impax EE, Agfa Health Care N.V., Mortsel, Belgium). Among various means of radiographical determination of the severity of knee OA, previous studies recommended the measurement of joint space width due to superior reliability and validity over other methods $[4,20]$. From weight-bearing flexed radiographs (Schuss-view) $[8,11,13]$, the location of the most pronounced narrowing of the joint space width was identified (Fig. 1). The joint space was measured to one decimal place of a millimetre at that point to determine the parameter 'joint space width (JSW)'. In the case of not only full joint space collapse but even bony defects (e.g. femoral condyle eroding in the tibia), JSW was defined as $0 \mathrm{~mm}$ because negative measurements would have been less accurate. The JSW measurements were rounded to full millimetres and patients were assigned to Group 1 if JSW was 0 or $1 \mathrm{~mm}$, and to Group 2 if JSW was $\geq 2 \mathrm{~mm}$.

For patient-reported outcome measurement, the Western Ontario and MacMaster Universities (WOMAC) Osteoarthritis Index score $[2,16]$ was analysed. The questionnaire was completed the day before surgery and again postoperatively 1 year after surgery. Implant survival as the second outcome parameter was extracted from the arthroplasty register of Tyrol.

Data analysis was performed with $\mathrm{R}$ version 3.6.3. (The $\mathrm{R}$ foundation for Statistical Computing, c/o Department of Statistics and Mathematics, University of Vienna, Vienna, Austria). The Mann-Whitney $U$ test was applied to test for significant differences between groups regarding the WOMAC

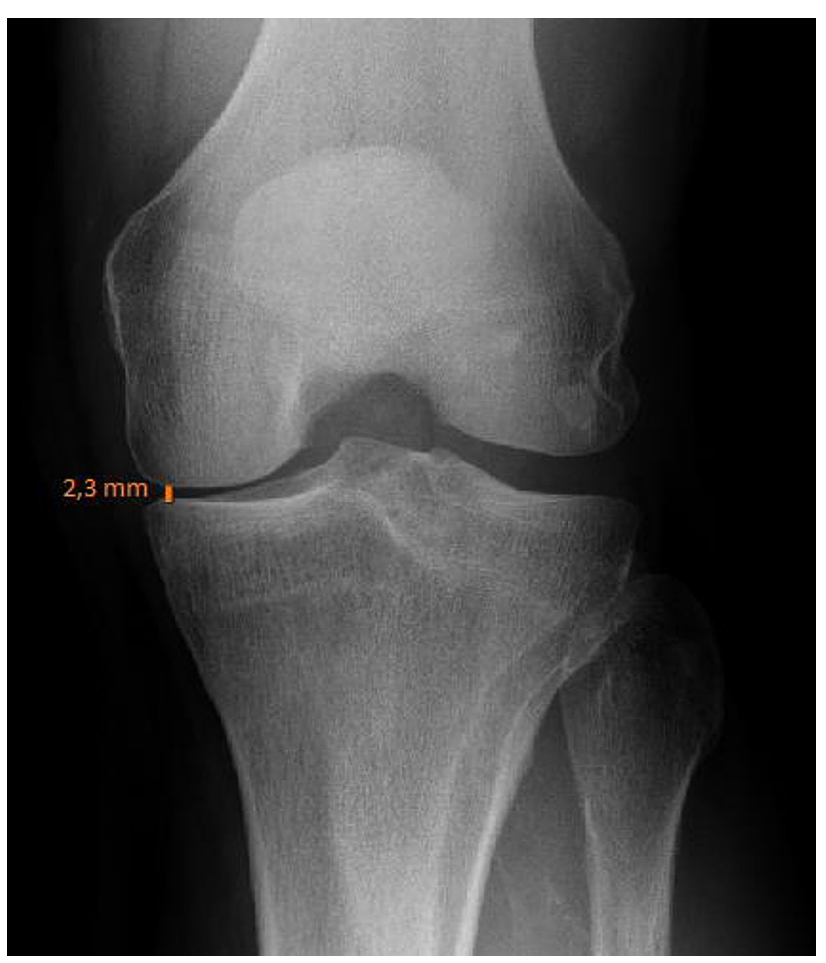

Fig. 1 Measurement of joint space width from weight-bearing Schuss-view radiographs 
total score and the WOMAC subscores. For Kaplan-Meier estimation, the R package survival version 3.1.8 was used. Differences in survival curves were tested using the log rank test. Alpha was defined as 0.05 (two-tailed).

\section{Results}

The two groups together comprised 150 patients after medial UKA (98 females, 52 males). Age was 66 years (Md, IQR 13) and BMI was $28 \mathrm{~kg} / \mathrm{m}^{2}$ (Md, IQR 5.9). JSW was $1 \mathrm{~mm}$ (Md, IQR 1, range $0-4 \mathrm{~mm}$ ). There were 80 patients in Group 1 (JSW 0-1 mm) (age 66, IQR 11, BMI 27.8) and 70 patients in Group 2 (JSW $\geq 2 \mathrm{~mm}$ ) (age 64, IQR 15, BMI

Table 1 WOMAC total scores and subscores at baseline and 1 year postoperative for the two groups

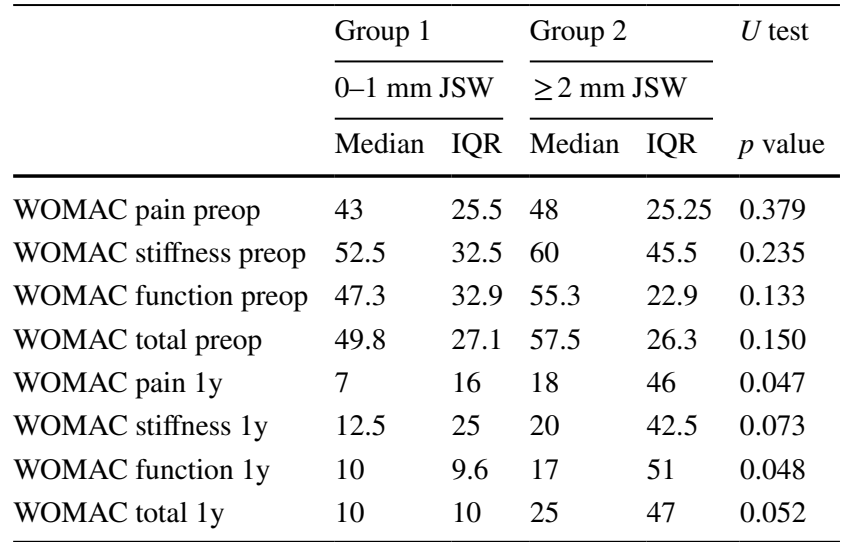

WOMAC Western Ontario and MacMaster Universities Osteoarthritis Index, $J S W$ Joint space width, $I Q R$ Inter-quartile range, preop preoperative, $y$ year, $U$ test Mann-Whitney $U$ test
28.1). In Group 2, mean JSW was $2.2 \mathrm{~mm} \pm 0.4 \mathrm{~mm}$ (SD, range $2-4 \mathrm{~mm}$ ). Preoperative WOMAC total and WOMAC subscores showed no significant differences between Group 1 and Group 2 (Table 1).

WOMAC pain and WOMAC function exhibited significantly poorer results in patients with $\geq 2 \mathrm{~mm}$ JSW. WOMAC pain at 1 year postoperative was $7 \pm 16$ in patients with $0-1 \mathrm{~mm}$ JSW and $18 \pm 46$ in patients with $\geq 2 \mathrm{~mm}$ JSW $(p=0.047)$. WOMAC function at 1 year postoperative was $10 \pm 9.6$ in patients with $0-1 \mathrm{~mm} \mathrm{JSW}$ and $17 \pm 51$ in patients with $\geq 2 \mathrm{~mm}$ JSW 1 year postoperative ( $p=0.048$, see Table 1 for full information on WOMAC data). Post hoc power analysis revealed a power of 0.91 .

Five year prosthesis survival was $92.3 \%$ in patients with $0-1 \mathrm{~mm} \mathrm{JSW}$ and $81.1 \%$ in patients with $\geq 2 \mathrm{~mm}$ JSW ( $p=0.016)$ (Fig. 2, Table 2). Post hoc power analysis revealed a power of 0.87 .

Table 2 Implant survival within 5 years postoperative for both groups

\begin{tabular}{lllll}
\hline & Year & Survival & Upper 95\% CI & Lower 95\% CI \\
\hline Group 1 (0-1 mm & $1 \mathrm{y}$ & 1.000 & 1.000 & 1.000 \\
JSW) & 2y & 0.950 & 0.903 & 0.999 \\
& 3y & 0.937 & 0.885 & 0.992 \\
& 4y & 0.923 & 0.866 & 0.984 \\
Group 2 ( $\geq 2 \mathrm{~mm}$ & 5y & 0.923 & 0.866 & 0.984 \\
JSW) & $1 \mathrm{y}$ & 0.929 & 0.870 & 0.991 \\
& 2y & 0.886 & 0.814 & 0.963 \\
& 3y & 0.857 & 0.779 & 0.943 \\
& $4 \mathrm{y}$ & 0.843 & 0.761 & 0.932 \\
& $5 \mathrm{y}$ & 0.811 & 0.723 & 0.909 \\
\hline
\end{tabular}

$J S W$ joint space width, $C I$ confidence interval

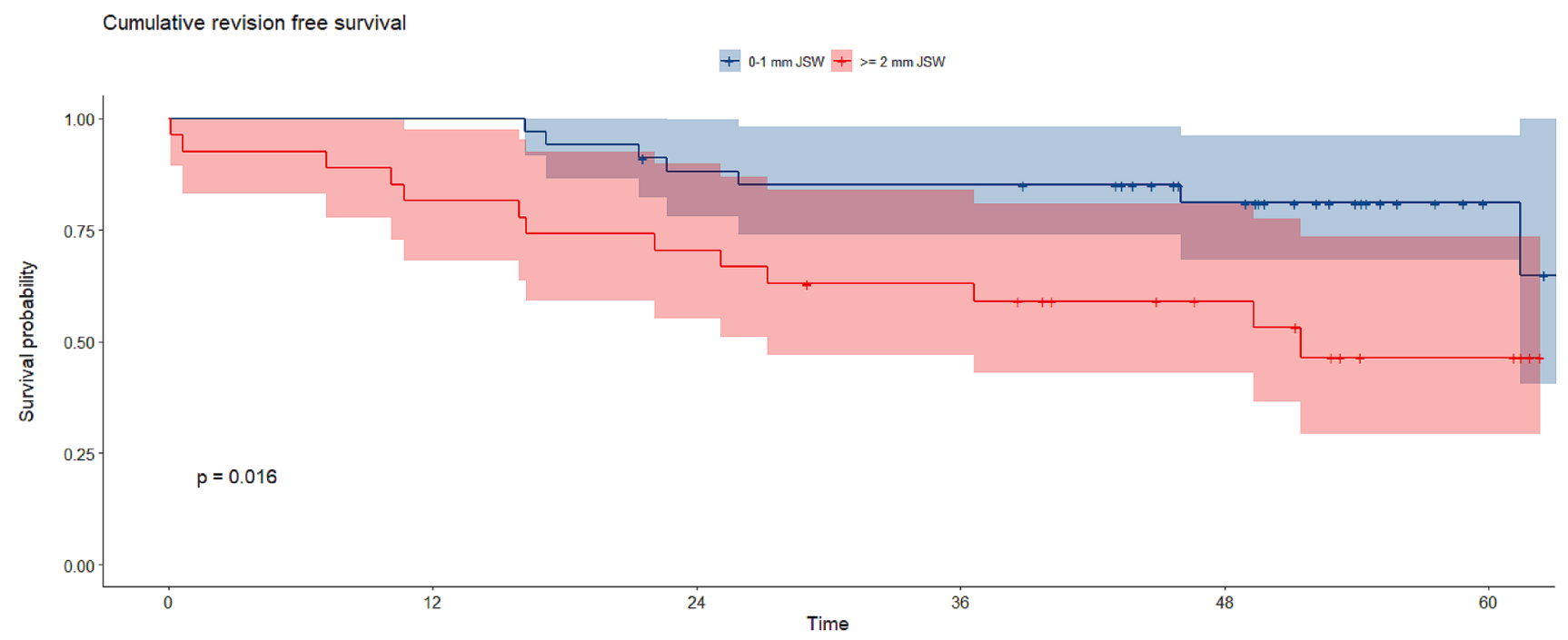

Fig. 2 Kaplan-Meyer estimator for implant survival within 5 years postoperative for both groups 


\section{Discussion}

The most important findings were the significantly poorer WOMAC pain and function scores in patients in Group 2 (mean JSW $2.4 \mathrm{~mm}$, range $2-4 \mathrm{~mm}$ ), although no such significant differences were observed for the WOMAC total score. Moreover, after a 5 year follow-up interval patients with a JSW of $\geq 2 \mathrm{~mm}$ revealed significantly inferior implant survival as compared to patients with $0-1 \mathrm{~mm}$ JSW.

When attempting to compare the findings of the current study with those reported in previous publications, it was seen that only three publications are available on the identical topic [5, 7, 10]. Knifsund et al. investigated 294 UKA cases [7]. They reported that those undergoing UKA with preoperative Kellgren-Lawrence (KL) Grade 0-2 had a significantly greater risk of later reoperation than did those with KL Grade 3-4. Knifsund et al. also stated that knees with a joint space width greater than $1 \mathrm{~mm}$ have a greater risk for revision surgery. The findings of the current study support the findings made in the study by Knifsund et al. However, Knifsund et al. did not publish patient-reported outcome parameters, as did the current study. Therefore, no comparisons are possible in this regard. Maier et al. also investigated the influence of the preoperative stage of OA on medial UKA outcome [10]. The authors reported on 64 patients and compared cases with partial joint space collapse and those with complete joint space taken from varus stress radiographs in $20^{\circ}$ knee flexion. Similar to the current study, Maier et al. analysed knee scores and implant survival. The authors reported that there were no significant differences between groups with regard to postoperative Oxford Knee Score, Knee Society Score or VAS pain. In addition, the authors also investigated 5 year implant survival and reported survival figures of $97 \%$ and $84 \%$ in the groups with complete and partial joint space collapse, respectively. Interestingly, the authors report a $\mathrm{p}$ of 0.095 , which might be an indicator for a beta error, especially when interpreted together with a relatively low case number of 32 per group. In other words, it can be speculated whether Maier et al. would have achieved statistical significance if they had had more cases. Unfortunately, no power analysis was provided by Maier et al. Hamilton et al. investigated 94 UKA with preoperatively only partial cartilage loss [5]. The 94 cases were 1:2 matched with 188 cases with full-thickness cartilage loss and compared with regard to Oxford Knee Score and Knee Society Scores. After 1, 2 and 5 years, the authors found significantly poorer scores in patients with preoperatively only partial cartilage loss. In this regard, the findings made by Hamilton et al. are congruent with the findings made in the current study. Hamilton et al. also analysed implant survival and reported no differences in implant survival between UKA patients with partial cartilage loss and those with full cartilage loss $(p=0.06)$. In this connection, the findings of the current study conflict with the findings made by Hamilton et al. However, the $p$ of 0.06 provided by Hamilton et al. again raises the suspicion of a beta error, especially because no power value was reported. Another potential explanation for the significantly poorer knee score results without inferior implant survival could be that Hamilton et al. were more conservative when it came to revision surgery. Interestingly, Hamilton et al. also investigated the reoperation rate (surgery without removal of any of the UKA components). In this connection, the authors found highly significant differences between the groups with a 5 year reoperation rate of $10.9 \%$ and $3.9 \%$ for patients with partial cartilage loss and full cartilage loss, respectively $(p<0.001)$.

When attempting to analyse for differences amongst the three previous studies, it is seen that only two studies investigated knee scores $[5,10]$. Of these two, only one study found a significantly poorer score outcome among patients with incomplete joint space collapse [5]. All three previous studies investigated implant survival. Only one of them found significantly poorer implant survival among patients with incomplete joint space collapse [7], while the other two reported a petween 0.05 and 0.1 , and therefore, potentially suffered from beta error $[5,10]$, especially because no power was stated.

In the TKA field, the situation seems to be clearer. Seven studies were published that examined the relationship between preoperative OA severity and clinical outcome [6, 9, 12, 14, 17-19]. In summary, the results favoured an association between preoperative OA grade and TKA outcome (five studies pro: two studies contra: the more preoperative degeneration, the better the TKA outcome. However, the TKA field does not support clear conclusions for UKA patients.

The following study limitations are acknowledged. First, it was a retrospective study with the typical weaknesses associated with such studies: selection bias, information bias, inability to investigate parameters other than those previously collected during clinical routine, reliance on data collected by others etc. Second, although previously suggested we did not succeed in collecting physical activity data or health-related quality of life data in conjunction with the knee-specific WOMAC data. Third, it was not possible to control for proper stratification of preoperative symptoms. As this was a retrospective study, it was just pure coincidence that the preoperative WOMAC scores were balanced between the groups. As the groups showed no differences in terms of WOMAC prior to surgery, this means surgical indication was made predominantly on the basis of clinical and subjective assessment and not radiography. 
It is regarded as a strength of our study that radiographic severity of knee OA was assessed in terms of JSW from weight-bearing radiographs, which was found to be the preferable method $[4,20]$. Another strength was that both a well-known outcome parameter (WOMAC) and the implant survival rate were investigated.

The study findings are regarded as having high clinical relevance. Particularly high patient satisfaction can be expected when using, complete joint space collapse' as indication for UKA surgery. In the case of incomplete joint space collapse, further conservative therapy $[1,3]$ or jointpreserving knee surgery (guided by MRI diagnostics) [15] might be considered an alternative to UKA.

\section{Conclusions}

Clinical outcome in patients with preoperative complete joint space collapse (0-1 mm JSW) was superior to that in patients with incomplete joint space collapse. This was true for both 1 year postoperative WOMAC pain and WOMAC function and for 5 year implant survival rates. Complete radiographic joint space collapse provides superior outcome after UKA as compared with partial joint space collapse.

Acknowledgements This study was conducted with internal funds received from the Dept. of Orthopaedics and Traumatology, Medical University of Innsbruck, Innsbruck, and the Dept. of Clinical Epidemiology, Tirol Kliniken, Innsbruck.

Author contributions During the processing of this study, AW was responsible for collecting and analysing the data as well as writing the manuscript. HL and BP were responsible for analysis of the data obtained and for writing the manuscript. AZ, MK and ML were responsible for analysing the statistical data, and for writing the manuscript. DD performed manuscript revision.

Funding No funding was received. Open access funding provided by University of Innsbruck and Medical University of Innsbruck.

\section{Declarations}

Conflict of interest There are no conflicts of interests to declare. There are no financial conflicts of interest to declare.

Ethical approval We confirm that any aspect of the work covered in this manuscript that has involved human patients has been conducted with the ethical approval (approval No.AN2017-0021-370/4.1) of all relevant bodies and that such approvals are acknowledged within the manuscript.

Open Access This article is licensed under a Creative Commons Attribution 4.0 International License, which permits use, sharing, adaptation, distribution and reproduction in any medium or format, as long as you give appropriate credit to the original author(s) and the source, provide a link to the Creative Commons licence, and indicate if changes were made. The images or other third party material in this article are included in the article's Creative Commons licence, unless indicated otherwise in a credit line to the material. If material is not included in the article's Creative Commons licence and your intended use is not permitted by statutory regulation or exceeds the permitted use, you will need to obtain permission directly from the copyright holder. To view a copy of this licence, visit http://creativecommons.org/licenses/by/4.0/.

\section{References}

1. Altmis H, Oskay D, Elbasan B, Duzgun I, Tuna Z (2018) Mobilization with movement and kinesio taping in knee arthritis-evaluation and outcomes. IntOrthop 42:2807-2815

2. Bellamy N, Buchanan WW, Goldsmith CH, Campbell J, Stitt LW (1988) Validation study of WOMAC: a health status instrument for measuring clinically important patient relevant outcomes to antirheumatic drug therapy in patients with osteoarthritis of the hip or knee. J Rheumatol 15:1833-1840

3. Campos ALS, EA RSP, da Silva EB, Fayad SG, Acerbi LD, de Almeida FN et al (2017) Viscosupplementation in patients with severe osteoarthritis of the knee: six month follow-up of a randomized, double-blind clinical trial. IntOrthop 41:2273-2280

4. Gossec L, Jordan JM, Mazzuca SA, Lam MA, Suarez-Almazor ME, Renner JB et al (2008) Comparative evaluation of three semi-quantitative radiographic grading techniques for knee osteoarthritis in terms of validity and reproducibility in 1759 X-rays: report of the OARSI-OMERACT task force. Osteoarthritis Cartilage 16:742-748

5. Hamilton TW, Pandit HG, Inabathula A, Ostlere SJ, Jenkins C, Mellon SJ et al (2017) Unsatisfactory outcomes following unicompartmental knee arthroplasty in patients with partial thickness cartilage loss: a medium-term follow-up. Bone Joint J 99-B:475-482

6. Keurentjes JC, Fiocco M, So-Osman C, Onstenk R, KoopmanVan Gemert AW, Poll RG et al (2013) Patients with severe radiographic osteoarthritis have a better prognosis in physical functioning after hip and knee replacement: a cohort-study. PLoS ONE 8:e59500

7. Knifsund J, Hatakka J, Keemu H, Makela K, Koivisto M, Niinimaki T (2017) Unicompartmental knee arthroplasties are performed on the patients with radiologically too mild osteoarthritis. Scand J Surg 106:338-341

8. Le Graverand MP, Vignon EP, Brandt KD, Mazzuca SA, Piperno M, Buck R et al (2008) Head-to-head comparison of the Lyon Schuss and fixed flexion radiographic techniques. Longterm reproducibility in normal knees and sensitivity to change in osteoarthritic knees. Ann Rheum Dis 67:1562-1566

9. Liebensteiner M, Wurm A, Gamper D, Oberaigner W, Dammerer D, Krismer M (2019) Patient satisfaction after total knee arthroplasty is better in patients with pre-operative complete joint space collapse. IntOrthop 43:1841-1847

10. Maier MW, Kuhs F, Streit MR, Schuhmacher P, Walker T, Ewerbeck V et al (2015) Unicompartmental knee arthroplasty in patients with full versus partial thickness cartilage loss (PTCL): equal in clinical outcome but with higher reoperation rate for patients with PTCL. Arch Orthop Trauma Surg 135:1169-1175

11. Mazzuca SA, Hellio Le Graverand MP, Vignon E, Hunter DJ, Jackson CG, Kraus VB et al (2008) Performance of a non-fluoroscopically assisted substitute for the Lyon schuss knee radiograph: quality and reproducibility of positioning and sensitivity to joint space narrowing in osteoarthritic knees. Osteoarthritis Cartilage 16:1555-1559

12. Merle-Vincent F, Couris CM, Schott AM, Conrozier T, Piperno M, Mathieu P et al (2011) Factors predicting patient satisfaction 
2 years after total knee arthroplasty for osteoarthritis. Joint Bone Spine 78:383-386

13. Piperno M, Hellio Le Graverand MP, Conrozier T, Bochu M, Mathieu P, Vignon E (1998) Quantitative evaluation of joint space width in femorotibial osteoarthritis: comparison of three radiographic views. Osteoarthritis Cartilage 6:252-259

14. Polkowski GG 2nd, Ruh EL, Barrack TN, Nunley RM, Barrack RL (2013) Is pain and dissatisfaction after TKA related to early-grade preoperative osteoarthritis? ClinOrthopRelat Res 471:162-168

15. Ruangsomboon $P$, Chareancholvanich $K$, Harnroongroj T, Pornrattanamaneewong C (2017) Survivorship of medial opening wedge high tibial osteotomy in the elderly: two to ten years of follow up. IntOrthop 41:2045-2052

16. Stucki G, Meier D, Stucki S, Michel BA, Tyndall AG, Dick W et al (1996) Evaluation of a German version of WOMAC (Western Ontario and McMaster Universities) arthrosis index. Z Rheumatol 55:40-49

17. Tilbury C, Holtslag MJ, Tordoir RL, Leichtenberg CS, Verdegaal SH, Kroon HM et al (2016) Outcome of total hip arthroplasty, but not of total knee arthroplasty, is related to the preoperative radiographic severity of osteoarthritis. A prospective cohort study of 573 patients. ActaOrthop 87:67-71

18. Valdes AM, Doherty SA, Zhang W, Muir KR, Maciewicz RA, Doherty M (2012) Inverse relationship between preoperative radiographic severity and postoperative pain in patients with osteoarthritis who have undergone total joint arthroplasty. Semin Arthritis Rheum 41:568-575

19. Vina ER, Hannon MJ, Kwoh CK (2016) Improvement following total knee replacement surgery: exploring preoperative symptoms and change in preoperative symptoms. Semin Arthritis Rheum 45:547-555

20. Wright RW, Group M (2014) Osteoarthritis classification scales: interobserver reliability and arthroscopic correlation. J Bone Joint Surg Am 96:1145-1151

Publisher's Note Springer Nature remains neutral with regard to jurisdictional claims in published maps and institutional affiliations. 